\title{
PCR dependent biases could significantly affect quantitative estimation of plant mix composition
}

\author{
Valeriia Kaptelova \\ Central Research Institute of \\ Epidemiology, Moscow, Russia \\ valeriia.kaptelova@gmail.com \\ Denis Omelchenko \\ Institute of Information Transmission \\ Problems, Moscow, Russia \\ omdeno@gmail.com \\ Andrey Ayginin \\ Center for Strategic Planning and \\ Management of Biomedical Health \\ Risks Moscow, Russia \\ ayginin75@gmail.com \\ Andrei Samoilov \\ Central Research Institute of \\ Epidemiology, Moscow, Russia \\ andrei.samoilov@gmail.com
}

\author{
Maria Logacheva \\ Skolkovo Institute of Science and \\ Technology, Skolkovo, Russia \\ Lomonosov Moscow State University, \\ Moscow, Russia \\ maria.log@gmail.com \\ Anna Fedotova \\ Skolkovo Institute of Science and \\ Technology, Skolkovo, Russia \\ Lomonosov Moscow State University, \\ Moscow, Russia \\ Kamil Khafizov \\ Center for Strategic Planning and \\ Management of Biomedical Health \\ Risks Moscow, Russia \\ kkhafizov@gmail.com
}

\author{
Anna Speranskaya \\ Central Research Institute of \\ Epidemiology, Moscow, Russia \\ Lomonosov Moscow State University, \\ Moscow, Russia \\ hanna.s.939@gmail.com \\ Anastasia Krinitsina \\ Lomonosov Moscow State University, \\ Moscow, Russia \\ I.M. Sechenov First Moscow State \\ Medical University, Pharmaceutical \\ Natural Science Department, \\ Moscow, Russia \\ ankrina@gmail.com \\ Elena Korneenko \\ Central Research Institute of \\ Epidemiology, Moscow, Russia \\ lennatta@yandex.ru
}

\begin{abstract}
Metagenomic analysis using high-throughput sequencing is an intensively developing approach nowadays. One of its problems is adequate quantification of components in metagenomic samples.
\end{abstract}

Keywords - food; nrITS1; metagenomics; polymerase; NGS

\section{Motivation and aim}

Metagenomic analysis using high-throughput sequencing is an intensively developing approach nowadays. In contrast to the qualitative analysis, the quantitative one still remains very challenging. The main factors that hamper quantitative analysis are differences in GC-content and length of marker regions. Our aim was to investigate and improve the potential of metabarcoding as analytic systems for quantitative identification of complex plant mixes used for food. In plants nrITS1 sequence length can vary almost twice. The percentage of the GC-composition also varies significantly: $\min 10 \% /$ median $/ \max 10 \%$ were found 48.1/58.0/67.5.

\section{Methods}

We prepared and analyzed the artificial plant mixes from leaves of seven cultivated plants used for food, as well as model species Arabidopsis thaliana. Plant species were chosen according to their different GC-composition (from 53\% to 72,9\%) and length of nrITS1 (from 192 to $348 \mathrm{bp}$ ) in order to be able to compare the results of the evaluation. The following species were used for the experiments: Oryza sativa L. (rice), Arabidopsis thaliana (L.) Heynh, Helianthus annuus L. (sunflower), Fagopyrum esculentum Moench (buckwheat), Cucurbita pepo L. (pumpkin). Equal weights of leaf powder were mixed, then DNA was extracted using sorbent purification-based Diamond DNA Plant kit (ABT, Russia). nrITS1 barcodes were amplified (in three replicates for each mix) using polymerases from different vendors: Encyclo proofreading polymerase («Encyclo Plus PCR kit», Evrogen, Russia) and Q5 High-Fidelity polymerase («Q5®) Hot Start High-Fidelity 2X Master Mix», NEB, England). Libraries were constructed using Ion Xpress $^{\mathrm{TM}}$ Plus Fragment Library Kit (Thermo Fisher Scientific) according to manufacturer's instructions, including amplification by Platinum HiFi Polymerase. Another approach was library preparation using the same kit but without amplification (PCR-free). Further all libraries were sequenced on Ion Torrent S5.

\section{Results}

For the libraries prepared from DNA samples with the standard protocol Ion Xpress Plus the congruence with expected results was extremely poor for the mixes containing $O$. sativa (Table 1). O. sativa ITS1 has high GC content $72,9 \%$. On the other hand, the nrITS1 of F. esculentum consists of $68,5 \% \mathrm{GC}$ (i.e. the value is also high) but analysis of samples containing this component showed correct results, perhaps due to shorter length of barcode (in comparing with nrITS1 of the other components in the same samples). We made a series of additional experiments using fragment length analysis of amplified libraries and showed that at least one of the reasons for bias is inefficient amplification of extremely high GC-rich fragments by Platinum HiFi polymerase during library preparation. When PCR-free amplification protocol was used the quantification, results were improved significantly for $O$. sativa containing mixes. The aim of this investigation was to check the hypothesis that GCcomposition and length of DNA-barcodes could significantly affect quantitative identification of multicomponent plant foods. We found that this dependence exists indeed and can affect PCR efficiency leading to yield inaccurate results of quantification. It may be possible to improve the interpretation of sequencing data using optimization of experimental protocols, additional controls [1] and through the implementation of special algorithms that correct sample preparation biases through correction coefficients.

\section{ACKNOWLEDGMENT}

Supported by the Ministry of education and science of Russia, project \# 14.609.21.0101, unique grant identifier RFMEFI60917X0101. 


\section{REFERENCES}

[1] D.O. Omelchenko, A.S. Speranskaya, A.A. Ayginin, K. Khafizov, A.A.

Krinitsina, A.A. Fedotova, D.V. Pozdyshev, V.Y. Shtratnikova, E.V. Kupriyanova, G.A. Shipulin, M.D. Logacheva. (2019) Improved Protocols of

TABLE 1

\begin{tabular}{|c|c|c|c|c|c|c|}
\hline \multirow[t]{2}{*}{$\begin{array}{c}\text { Components of } \\
\text { mix }\end{array}$} & \multirow[t]{2}{*}{$\begin{array}{c}\text { GC- } \\
\text { composition } \\
(\%)\end{array}$} & \multirow[t]{2}{*}{$\begin{array}{l}\text { Length } \\
\text { (b.p.) }\end{array}$} & $\begin{array}{c}\text { Q5 High- } \\
\text { Fidelity Hot } \\
\text { Start } \\
\text { polymerase, } \\
\% \text { of reads } \\
\end{array}$ & $\begin{array}{c}\text { Encyclo } \\
\text { proofreading } \\
\text { polymerase, \% } \\
\text { of reads }\end{array}$ & $\begin{array}{c}\text { Q5 High-Fidelity } \\
\text { Hot Start } \\
\text { polymerase, \% } \\
\text { of reads (PCR- } \\
\text { free) } \\
\end{array}$ & $\begin{array}{c}\text { Encyclo } \\
\text { proofreading } \\
\text { polymerase, \% of } \\
\text { reads (PCR-free) }\end{array}$ \\
\hline & & & Average (SD) & Average (SD) & Average (SD) & Average (SD) \\
\hline \multirow[t]{2}{*}{ A. thaliana } & 55,9 & 348 & $25,8(4,61)$ & $24,87(10,9)$ & $28,1(3,12)$ & $33,5(6,41)$ \\
\hline & 53 & 192 & $74,14(4,61)$ & $68,27(1,15)$ & $71,73(2,93)$ & $66,5(6,41)$ \\
\hline A. thaliana & 55,9 & 348 & $43,76(6,28)$ & $\mathrm{N} / \mathrm{A}$ & $45,27(2,02)$ & $57,63(0,81)$ \\
\hline F. esculentum & 68,5 & 259 & $56,09(6,32)$ & $\mathrm{N} / \mathrm{A}$ & $54,57(2,08)$ & $42,4(0,87)$ \\
\hline \multirow{2}{*}{$\begin{array}{c}\text { A. thaliana } \\
\text { O. sativa }\end{array}$} & 55,9 & 348 & $98,3(0,45)$ & $99,53(0,15)$ & $58,33(8,58)$ & $78,43(13,21)$ \\
\hline & 72,9 & 275 & $1,14(0,59)$ & $0,47(0,15)$ & $41,5(8,42)$ & $21,5(13,17)$ \\
\hline \multirow{3}{*}{$\begin{array}{c}\text { H. annuиs } \\
\text { F. esculentum }\end{array}$} & 50 & 343 & $62,61(6,86)$ & $55,17(6,78)$ & $58,67(5,13)$ & $63,43(15,72)$ \\
\hline & 68,5 & 259 & $37,09(7,28)$ & $44,77(6,9)$ & $40,45(4,85)$ & $36,57(15,72)$ \\
\hline & 59,5 & 300 & $8,85(11,73)$ & $42,07(2,5)$ & $20,07(17,44)$ & $\mathrm{N} / \mathrm{A}$ \\
\hline
\end{tabular}

ITS1-Based Metabarcoding and Their Application in the Analysis of Plant-Containing Products. Genes. 10(2): 122 